\title{
Novel technique for identification of the pulmonary intersegmental plane using manual jet ventilation during pulmonary segmentectomy
}

\author{
Shima Taguchi ${ }^{1}$, Noboru Saeki ${ }^{1}$, Atsushi Morio ${ }^{1}$, Ryuji Nakamura ${ }^{1}$, Satoshi Kamiya ${ }^{1}$, Tsuyoshi Ikeda ${ }^{1}$, Kyoko Oshita $^{1}$, \\ Hiroshi Hamada ${ }^{1}$, Yoshihiro Miyata ${ }^{2}$, Morihito Okada ${ }^{2}$, Yasuo M. Tsutsumi ${ }^{1}$ \\ ${ }^{1}$ Department of Anesthesiology and Critical Care, Hiroshima University, Hiroshima, Japan \\ 2Department of Surgical Oncology, Research Institute for Radiation Biology and Medicine, Hiroshima University, Hiroshima, Japan
}

Videosurgery Miniinv 2021; 16 (1): 169-174

DOI: https://doi.org/10.5114/wiitm.2020.95919

\begin{abstract}
Introduction: For successful pulmonary segmentectomy, the identification of boundaries between segments is important. Previous measures include tracing the intersegmental vessels by staining with a dye via the affected pulmonary artery or bronchus and inflating with oxygen via a high frequency ventilator. However, problems with these methods have been reported.

Aim: We developed a novel method using a manual jet ventilator (MJV) and investigated its efficacy in identification of the pulmonary intersegmental plane.

Material and methods: Patients underwent MJV for pulmonary segmentectomy in the period from January 2013 to December 2017 at our institution. The patients' characteristics, resected segments, availability of clear resection planes, and complications associated with MJV from medical records were investigated. A questionnaire survey was conducted with the surgeons on the effectiveness of lung segment identification using MJV.

Results: Of 199 cases of planned pulmonary segmentectomy, 171 cases with descriptions of identified intersegmental planes were analyzed. Of these, 152 (89\%) cases showed a clear boundary. There were 19 cases where the exact boundaries were not clearly identified, but segmentectomy was still performed. Furthermore, we found that identification of the right upper lobes was difficult $(p=0.0028)$. A subjective questionnaire was answered by the 12 surgeons who performed the procedures. All 12 responded that MJV was very effective or effective regarding clarity, safety, shorter identification time, and shorter resection time.

Conclusions: MJV enabled surgeons to more easily and safely identify the pulmonary intersegmental plane, thereby suggesting that MJV has clinical significance during pulmonary segmentectomy.
\end{abstract}

Key words: pulmonary segmentectomy, manual jet ventilation, fiberoptic bronchoscope, identification of the intersegmental plane.

\section{Introduction}

Recent advances in the treatment of lung cancer include detection of early stage, small-sized lung cancers combined with minimally invasive and lung function-sparing surgery. Of note, the outcomes of segmentectomy have been reported to be comparable with those of lobectomy [1, 2]. For successful segmentectomy, the ability to identify boundaries 
between the resected segment and others is crucial. However, there are many variations of intersegmental vessels, and the boundaries are not clear; therefore, a simple trace of the intersegmental vessels is not possible.

As a result, various techniques for identification have been reported including labeling the target segment with a dye via the pulmonary artery, bronchus, or peripheral vein, or inflating the segment with oxygen or air into the bronchus via the surgical field or fiberoptic bronchoscope (FOB) using a high frequency jet ventilator [3-12]. However, many of these techniques contain problems or difficulties including the potential for an air embolism and requiring a darkened environment.

Recently, Oizumi et al. reported the use of multi-detector computed tomography (CT) for segmentectomy [12]. This resulted in successful identification in $98 \%$ of the patients scheduled for segmentectomy. However, this method requires time for a knot to be produced in the operative field, as well as facilities that have high-resolution CT. By using a manual jet ventilator (MJV), we were able to insufflate the target lung segment with oxygen, thereby allowing for simpler, safer, and clearer identification of boundaries.

\section{Aim}

In this study, we retrospectively investigated the efficacy of this novel technique for detection of an intra-segmental border during surgery.

\section{Material and methods}

Patients scheduled for lung segmentectomy or subsegmentectomy using MJV (MCS-3, NIPPON MEGACARE CORPORATION, Tokyo, Japan) for identification of the intersegmental plane in our hospital

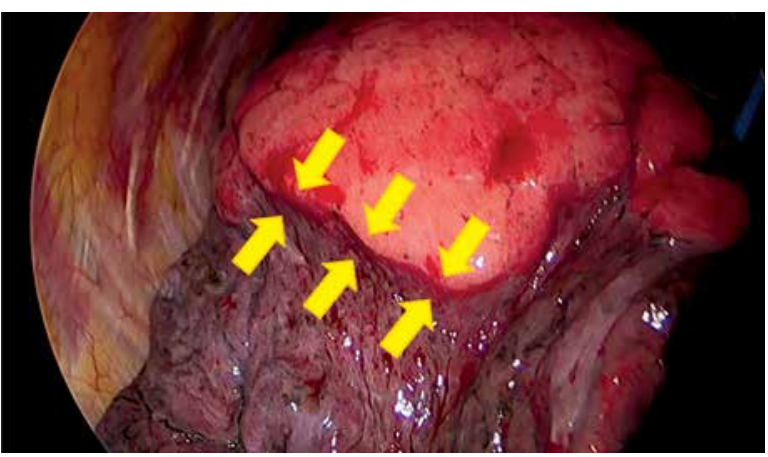

Photo 1. Intersegmental line were enrolled between January 1, 2013 and December 31, 2017. We excluded cases where identification by MJV was not necessary. We identified patients' characteristics including age, gender, height, body weight, and resected segments, availability of clear resection planes acquisition, and complications associated with MJV via patient medical records. We examined the relationship between success rate and patients' characteristics or resected segments.

\section{Manual jet ventilator}

The procedure for detecting the border between segments using MJV was as follows. We inserted the FOB into the bronchus of the lung segment to be resected from a double lumen tracheal tube (Broncho-Cath, Covidien Japan). Next, we inserted and fitted the tip of the MJV into the FOB air supply port, and the pressure was set at $0.14-0.21 \mathrm{MPa}$, with manual insufflation using oxygen into the resected segment while checking the expansion of the segment from the surgical field by the endoscopic image. Once we identified a clear intersegmental plane (Photo 1), the FOB was removed and the surgeon proceeded with the surgery.

\section{Questionnaire for surgeons}

A subjective questionnaire was given to the $12 \mathrm{ex}$ perienced surgeons who performed all of the procedures to compare the lung segment identification by MJV with other identification methods. The questionnaire included items related to clarity, simplicity, safety, shorter identification time, and shorter resection time. Each item was evaluated as very effective, effective, somewhat effective, or invalid.

\section{Statistical analysis}

Statistical analyses were carried out by unpaired Student's $t$ test, Fisher's exact test, and the $\chi^{2}$ test. A $p<0.05$ was considered statistically significant.

\section{Results}

Pulmonary segmentectomy was identified in 199 cases. Eighteen cases were excluded because we could not determine whether MJV was performed. In 10 cases, identification by MJV was no longer needed because of bronchial obstruction by the tumor or changes to the surgical procedures. Finally, 171 cases were analyzed (Figure 1). Table I lists 
the characteristics of the 171 patients (mean \pm SD). The success rates of identification for males and females were $85 \%$ and $93 \%$, respectively $(p=0.09)$, and there were no statistically significant differences in identification of the pulmonary intersegmental plane during segmentectomy with respect to patient characteristics.

Table II and Figure 2 show segments or subsegments scheduled for resection (right upper lobes, 35; right lower lobes, 48; left upper lobes, 53; left lower lobes, 35 cases); these included 15 subsegment cases. Clear planes were obtained in 152 (89\%) cases by MJV. In 19 cases, the resection borders could not be clearly identified in the following segments: right upper lobes in 10 cases $\left(S^{1}: 3, S^{2}: 4, S^{1}+S^{2}: 1, S^{1} a+\right.$ $\left.S^{2}: 1, S^{1} b+S^{2}: 1\right)$, right lower lobe in 2 cases $\left(S^{7}, S^{9}\right.$ $\left.+\mathrm{S}^{10}\right)$, left upper lobe in 4 cases $\left(\mathrm{S}^{1+2}: 1, \mathrm{~S}^{3}: 1, \mathrm{~S}^{1+2}+\right.$ $\left.S^{3}: 2\right)$, and left lower lobes in 3 cases $\left(S^{6}, S^{8}+S^{9}+\right.$ $\mathrm{S}^{10}, \mathrm{~S}^{9}+\mathrm{S}^{10}$ ) (Table III, Figure 2). Successful identifi-

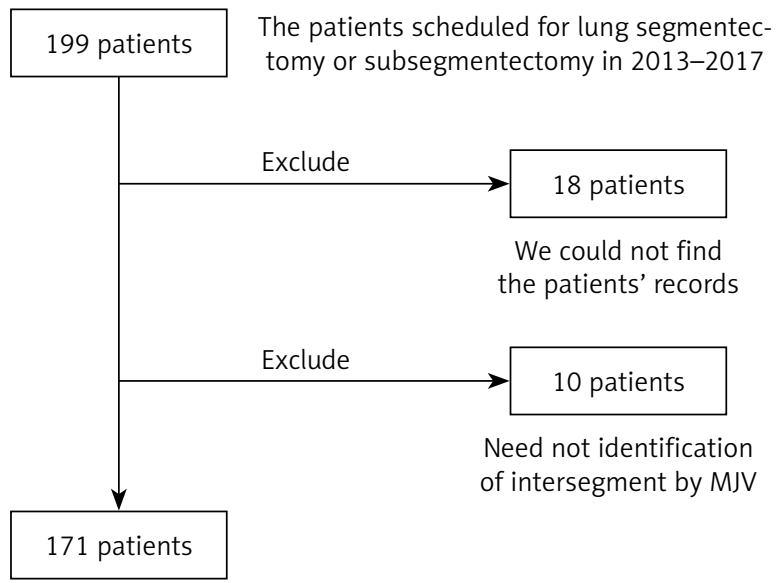

Figure 1. Flow chart showing examination cases

cation was significantly lower in the right upper lobe $(p=0.0028)$. There were no complications associated with MJV.

Table I. Patient characteristics $(n=171)$

\begin{tabular}{|lccccc|}
\hline Parameter & Total & \multicolumn{3}{c|}{ Identification } \\
\cline { 3 - 6 } & & Clear & Unclear & Success rate (\%) & $P$-value \\
\hline Age [years] & $67 \pm 10$ & $68 \pm 10$ & $67 \pm 11$ & 0.72 \\
\hline Patients: & 171 & 152 & 19 & 89 & 0.09 \\
\hline Male & 85 & 72 & 13 & 93 & 0.07 \\
\hline Female & 86 & 80 & 6 & & 0.80 \\
\hline Height $[\mathrm{cm}]$ & $159 \pm 9$ & $159 \pm 9$ & $163 \pm 9$ & $59 \pm 11$ & \\
\hline Weight $[\mathrm{kg}]$ & $58 \pm 11$ & $58 \pm 11$ & 59 & & \\
\hline
\end{tabular}

Mean $\pm S D, p$-values were analyzed by unpaired Student's t test and Fisher's exact test.

Table II. Area scheduled for resection $(n=171)$

\begin{tabular}{|c|}
\hline Right upper lobe: 35 \\
\hline$S^{1}: 12, S^{2}: 6, S^{1}+S^{2}: 4, S^{1} a+S^{2}: 1, S^{1} b+S^{2}: 1, S^{1}+S^{3}: 3, S^{1} b+S^{3}: 1, S^{2} b+S^{3} a: 1, S^{3}: 5$ \\
\hline Right lower lobe: 48 \\
\hline $\begin{array}{l}S^{6}: 17, S^{6} b+c: 1, S^{6}+S^{8} a: 3, S^{6}+S^{8}+S^{9}: 1, S^{6}+S^{9}+S^{10}: 1, S^{6}+S^{10} a+b: 1, S^{6}+S^{10} a: 1, S^{6}+S^{10}: 1, \\
S^{7}: 3, S^{7}+S^{8}+S^{9}: 1, S^{7}+S^{8}+S^{9}+S^{10}: 1, S^{8}: 5, S^{9}: 2, S^{8}+S^{9}: 2, S^{8}+S^{9} a: 2, S^{9}+S^{10}: 5, S^{10}: 1\end{array}$ \\
\hline Left upper lobe: 53 \\
\hline$S^{1+2}: 13, S^{1+2} a+b: 1, S^{3}: 6, S^{1+2}+S^{3} c: 1, S^{1+2}+S^{3}: 25, S^{4}+S^{5}: 7$ \\
\hline Left lower lobe: 35 \\
\hline$S^{6}: 18, S^{6}+S^{10}: 1, S^{8}: 4, S^{8}+S^{9}: 2, S^{8}+S^{9}+S^{10}: 3, S^{8}+S^{9}+S^{10} b c: 1, S^{9}+S^{10}: 3, S^{10}: 2$ \\
\hline Left lower lobe: 1 (needed to identify between lobes because it is unclear congenitally) \\
\hline
\end{tabular}

Right middle lobe $\left(S^{4}\right.$ or $\left.S^{5}\right): 0$ case. 


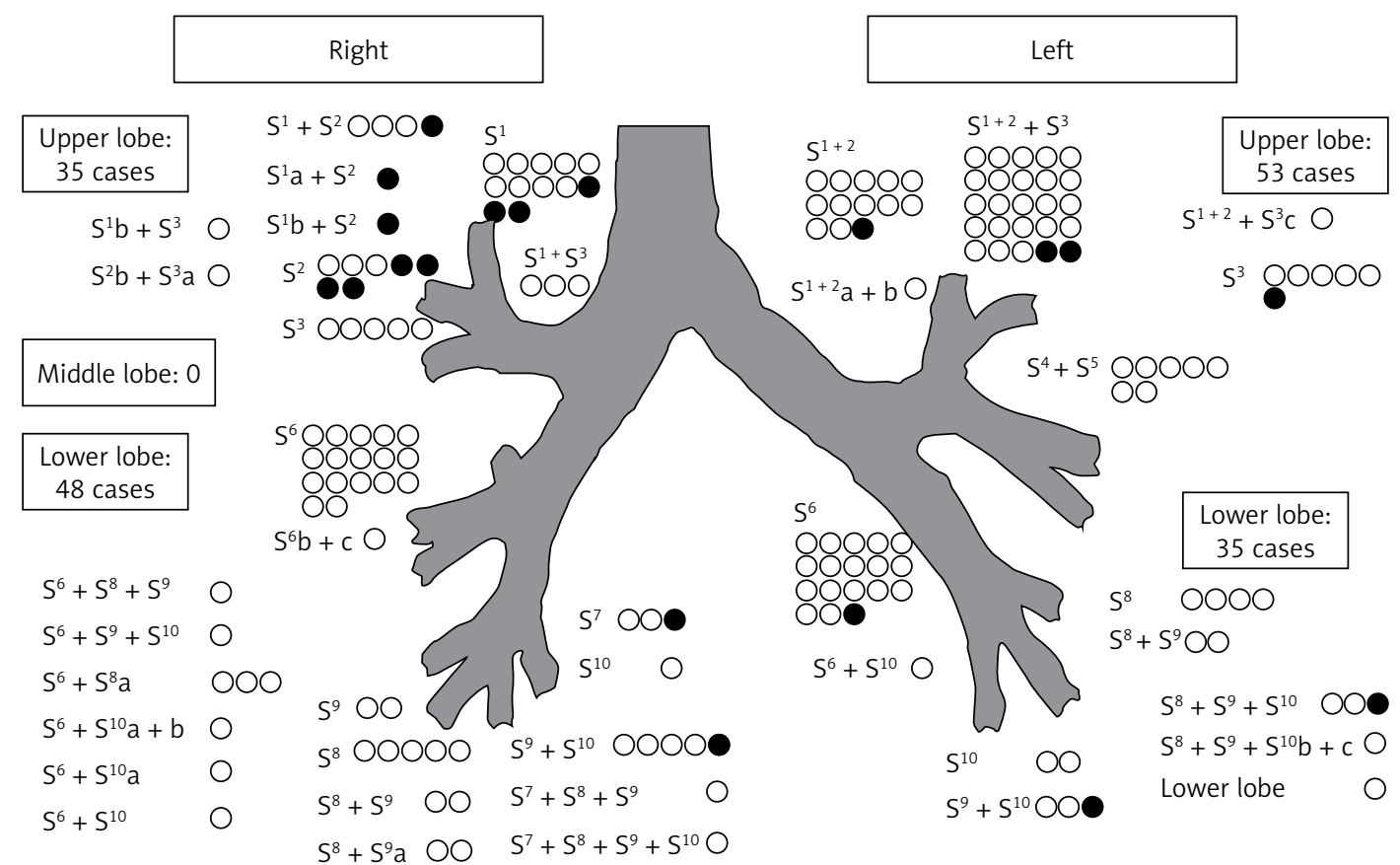

Figure 2. Area scheduled for resection and unclearly identification cases (filled points inside)

Table III. Unclear identification cases (19/171)

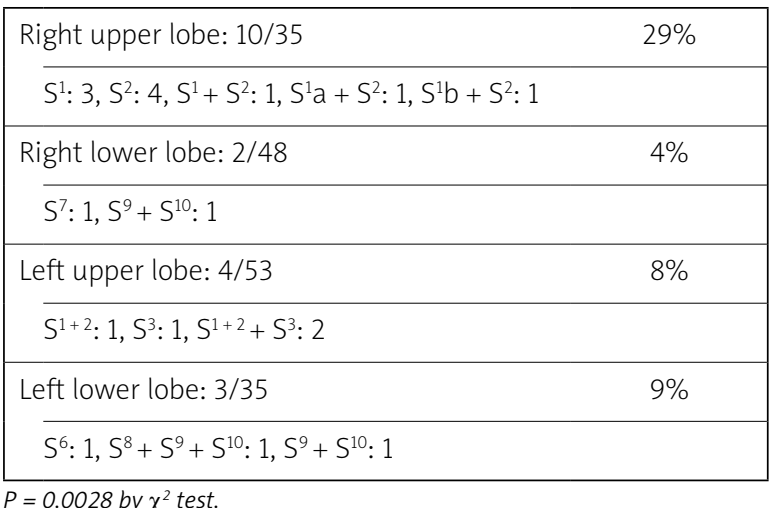

Our subjective questionnaire was answered by 12 experienced surgeons who performed these procedures. All 12 responded that MJV was "very effective" or "effective" with respect to clarity, safety, shorter identification time, and shorter resection time. Furthermore, 11 surgeons answered "very effective" or "effective" with regard to simplicity, and 1 surgeon replied "somewhat effective." None of the surgeons answered "invalid" for any items (Figure 3).

\section{Discussion}

The findings of this retrospective study revealed that a clear boundary was obtained in $89 \%$ of the patients who underwent MJV for planned pulmonary segmentectomy, whereas exact boundaries could not be identified in $11 \%$ of the cases. We also found that identification of the right upper lobes was difficult for segmentectomy. Additionally, surgeons preferred MJV via questionnaire (clarity, simplicity, safety, identification time, and resection time). These results suggest that MJV could be a safe and convenient procedure to identify the pulmonary intersegmental plane for segmentectomy.

There are many methods available for identification of lung segments. Sakamoto et al. used the temperature differences between the segments following ligation of the intersegmental vessels [3]; however, this technique has limitations as it is difficult to identify internal boundaries of the lung because only the external lung surface is examined using this method, making it difficult to determine in smaller incisions. Techniques using dyes such as methylene blue or indocyanine green have also been reported [4-7]. This method requires a dye to be injected directly into the resected bronchi $[4,5]$ or directly into the bronchial artery of the diseased segment to identify the target segments. Alternatively, the dye can be injected into the peripheral vein following ligation of the resected bronchial artery to identify the intended segments [6]. 


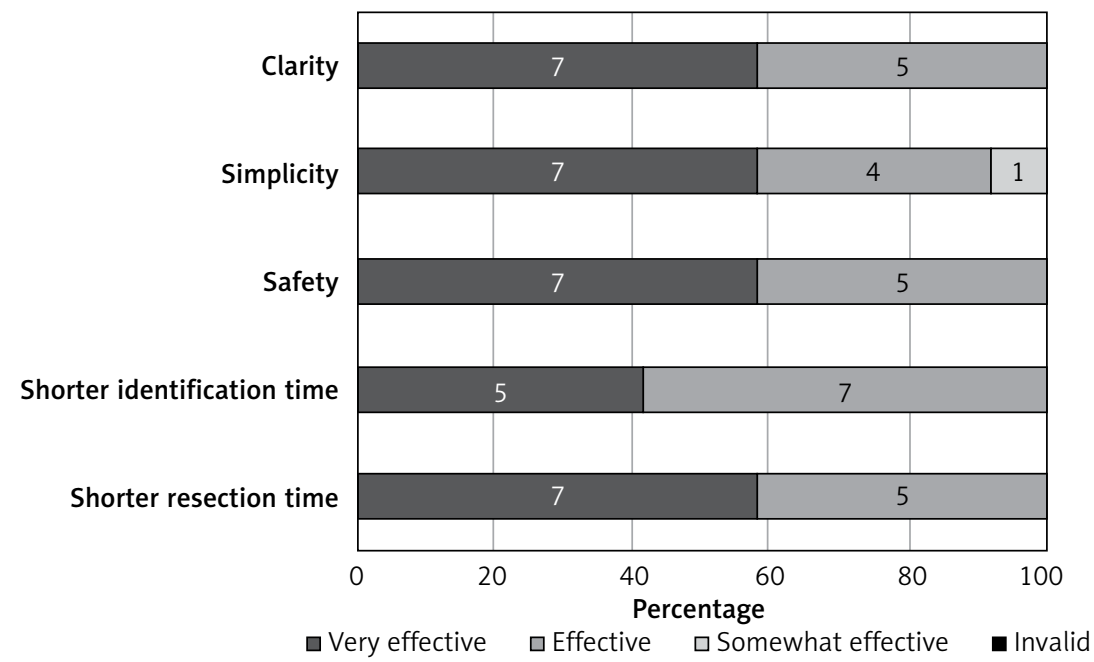

Figure 3. Questionnaire results for surgeons $(n=12)$

Zhang et al. reported that methylene blue is safer than indocyanine green because it results in a lower risk of liver damage and anaphylaxis and because the artery is softer and easier to pierce than the bronchus [4]; however, the procedure for dye injection is difficult as dye is injected directly into the surgical field over 1-2 min. Furthermore, this may change the volume of the segment and the dye may not spread consistently and evenly. Intravascular dye injection is easy to administer and results in small changes in the lung volume, and various studies have also reported the use of fluorescence $[6,7]$; however, these methods require a dark operation room for the use of an infrared thoracoscopy system, and the observable time is only 160-270 s. Furthermore, if any dye leaks into other areas by accident, the identification process becomes difficult, which can result in a reported identification rate of $84.6 \%$ [6].

Oxygen or air can be used to directly identify target segments. This method can be used to identify deflated or inflated segments, and revisions to the surgical procedure can be performed by suction. Oxygen can be introduced via two methods: via a cannulation into the segmental bronchi directly from the surgery field, or insertion via the FOB into the bronchus from a double lumen tracheal tube. However, oxygen may be accidentally injected intravenously, resulting in an air embolism. Therefore, introducing oxygen directly using the FOB is safer.

Previous studies have reported the inflation of the lung using an FOB as well as the use of a high frequency jet ventilator (HFV) $[8,9]$. Low flow oxygen rates (approximately $1-3 \mathrm{l} / \mathrm{min}$ ) resulted in leaks from the gap of the FOB, and the segment failed to expand sufficiently. However, it is difficult to administer oxygen at a high flow rate due to sudden bolus changes. Similarly, HFV has been reported to cause sudden expansion of the segment by blowing air with HFV after insertion of an FOB, allowing for fine tuning of the pressures [9]. An HFV starting at a low pressure before the FOB insertion and followed by a gradual rise in pressure after reaching the target segment makes it difficult to fine tune the timing and duration of the oxygen supply.

Our technique uses MJV through the FOB to directly inflate the resected lung to identify the lung intersegmental plane. MJV is smaller than HFV, and it can be easily moved because it can be carried on the fiber light source device. Additionally, MJV can be used to easily adjust the inflation time as well as the amount of aeration in real time. Furthermore, a previous study reported that Kohn's pores, which are traffic branches between alveoli, are closed between segments, preventing inflation of the next segment [10]. We were able to obtain clear intersegmental planes by MJV in 152 of 171 cases (89\%), and the success rate was higher than those of previous studies that used a dye [6].

Finally, segmentectomy was possible in all 19 cases with unclear planes owing to a combination of identification methods by following vessels and using MJV. No complications were documented from MJV or FOB, such as bronchial damage, baro-trauma, or air embo- 
lism. Additionally, subjectively, all of our surgeons felt that MJV was safer, quicker, faster, and easier to help identify surgical margins, allowing for quicker identification of segmental planes, although other identifying methods were not used in our institute

There were some limitations to our study due to its retrospective nature. First, clear identification could not be achieved via MJV in 19 cases. Furthermore, 10 of these 19 cases were segments of the right upper lobe, and the success rate of identification was significantly low. These results may be due to the difficulty in induction of the FOB to the right upper lobe, especially, when the angle of the branch of the right upper lobe becomes steeper, the patient's height is higher and the distance from the hilum to the branch of the right upper lobe is longer. In addition, it might be more difficult to insert the FOB due to the surgical procedure as the surgeon may shift, constrict, and rotate the bronchi during the operation.

\section{Conclusions}

The lung area identification method by MJV is safe, convenient, and preferred by our surgeons to identify the pulmonary intersegmental plane. This method may have important clinical significance for similar future cases.

\section{Conflict of interest}

The authors declare no conflict of interest.

\section{References}

1. Okada M, Mimae T, Tsutani Y, et al. Segmentectomy versus lobectomy for clinical stage IA lung adenocarcinoma. Ann Cardiothorac Surg 2014; 3: 153-9.

2. Tsutani Y, Miyata Y, Nakayama H, et al. Oncologic outcomes of segmentectomy compared with lobectomy for clinical stage IA lung adenocarcinoma: propensity score-matched analysis in a multicenter study. J Thorac Cardiovasc Surg 2013; 146: 358-64.

3. Sakamoto K, Kanzaki M, Mitsuboshi S, et al. A novel and simple method for identifying the lung intersegmental plane using thermography. Interact Cardiovasc Thorac Surg 2016; 23: 171-3.

4. Zhang Z, Liao Y, Ai B, Liu C. Methylene blue staining: a new technique for identifying intersegmental planes in anatomic segmentectomy. Ann Thorac Surg 2015; 99: 238-42.

5. Oh S, Suzuki K, Miyasaka Y, et al. New technique for lung segmentectomy using indocyanine green injection. Ann Thorac Surg 2013; 95: 2188-90.

6. Tarumi S, Misaki N, Kasai Y, et al. Clinical trial of video-assisted thoracoscopic segmentectomy using infrared thoracoscopy with indocyanine green. Eur J Cardiothorac Surg 2014; 46: 112-5.
7. Oh Y, Quan YH, Kim M, et al. Intraoperative fluorescence image-guided pulmonary segmentectomy. J Surg Res 2015; 199 : 287-93.

8. Okada M, Mimura T, Ikegaki J, et al. A novel video-assisted anatomic segmentectomy technique: selective segmental inflation via bronchofiberoptic jet followed by cautery cutting. J Thorac Cardiovasc Surg 2007; 133: 753-8.

9. Koga H, Shitomi R, Shingu C, Noguchi T. New technique for the identification of the pulmonary segment using high frequency jet ventilation and bronchofiberscopy. Masui 2008; 57: 895-6.

10. Zuo Y, Li L, LiU S. Kohn's pore are not responsible for collateral ventilation between inflated and deflated segments: a microscopic study of pulmonary intersegmental septa in the human lung. J Anat 2015; 226: 381-5.

11. Oizumi H, Kato H, Endoh M, et al. Slip knot bronchial ligatation method for thoracoscopic lung segmentectomy. Ann Thorac Surg 2014; 97: 1456-8.

12. Oizumi H, Kato H, Endoh M, et al. Techniques to define segmental anatomy during segmentectomy. Ann Cardiothorac Surg 2014; 3: 170-5.

Received: 18.03.2020, accepted: 12.05.2020. 